\section{Performance limitation of subband adaptive equalisers}

\section{H. Mohamad, S. Weiss, M. Rupp and L. Hanzo}

The aim of this reported work was identifying and quantifying limitation for the subband adaptive equalisers. The derived Wiener solution is compared against simulations for the subband equaliser, and it is verified that aliasing caused in the subband decimation can be considered equivalent to channel noise. Finally, how this limitation can be mitigated by careful system design is discussed.

Introduction: Adaptive equalisers have been widely employed to mitigate the effect of intersymbol interference (ISI) and to retrieve a transmitted signal $u[n]$ from a distorted received copy $x[n]$ at the output of a channel $c[n]$ in the presence of channel noise $v[n]$, whereby Fig. 1 illustrates a general block diagram for channel equalisation [1] For a minimum mean square equaliser (MMSE), adaptive algorithms can be employed to adapt the equaliser $w[n]$ by minimising the error between the received signal $y[n]$ and the training sequence $d[n]$.

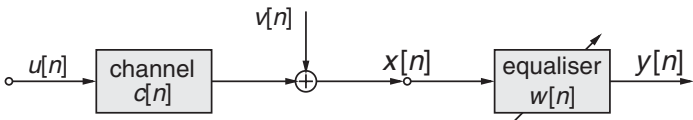

Fig. 1 Block diagram with channel impulse response $c[n]$, channel noise $v[n]$, and equaliser $w[n]$

Subband adaptive equaliser: An adaptive equaliser based on the subband decomposition method has been demonstrated to exhibit fast convergence speed at low computational complexity when long equalisers $w[n]$ are employed using the LMS-type algorithm [2, 3]. In related adaptive filtering application, subband techniques were previously proposed for echo cancellation applications [4] where the achievable MMSE performance has a lower limit imposed by the employed filter bank structure through aliasing in the subbands [5]. The schematic of a subband adaptive equaliser is shown in Fig. 2. Oversampled modulated filter banks are used for subband adaptive equalisation such that aliasing is restricted to the stopband of the analysis filters and can therefore be controlled by appropriate filter bank design [5]. Analysis filters $h_{k}[n]$ and sysnthesis filters $g_{k}[n]$ are derived from a real-valued lowpass prototype FIR filter $p[n]$ by using a generalised discrete Fourier transform (GDFT).

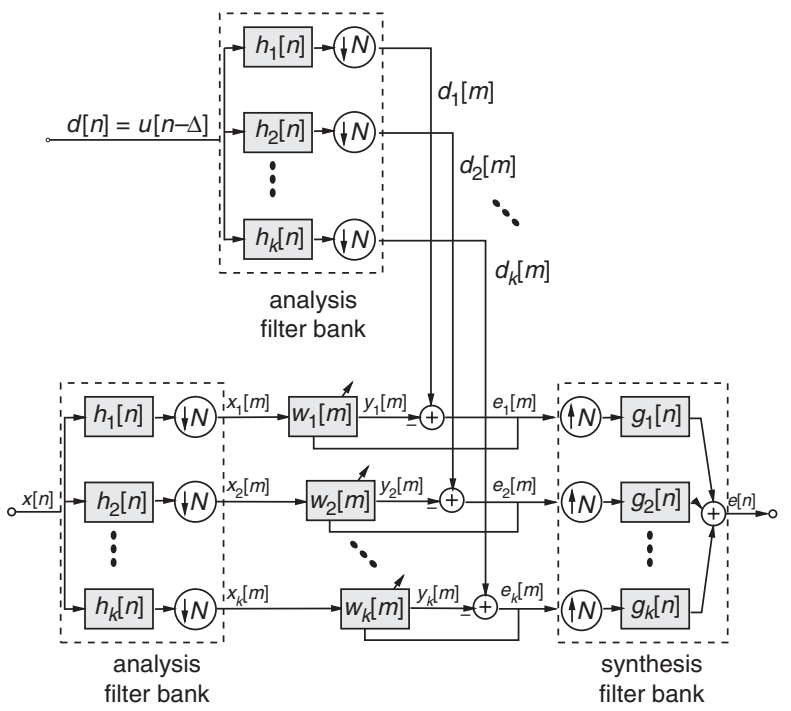

Fig. 2 Subband adaptive equaliser structure

Wiener solution: Assuming that the subband system employed perfectreconstruction (PR) filter banks, discussion on subband equaliser limitations owing to the aliasing problem is presented. The Wiener solution for fullband and subband equalisers is derived in order to investigate the effect of aliasing on the optimum MMSE solution.

The fullband equaliser's ability to combat ISI is limited by the channel noise $v[n]$ [1]. In reference to Fig. 1, applying the Wiener estimation of the transmitted signal $u[n]$ based on the noisy measurement $x[n]$, the optimal MMSE solution to $w[n]$ is given by the Wiener filter in the frequency domain [6] as

$$
W_{\text {opt }}\left(e^{j \Omega}\right)=\frac{P_{u u}\left(e^{j \Omega}\right) \cdot C^{*}\left(e^{j \Omega}\right)}{P_{u u}\left(e^{j \Omega}\right) \cdot\left|C\left(e^{j \Omega}\right)\right|^{2}+P_{v v}\left(e^{j \Omega}\right)}
$$

This assumes that $u[n]$ and $v[n]$ are statistically independent. In our notation, $P_{u u}\left(e^{j \Omega}\right)$ and $P_{v v}\left(e^{j \Omega}\right)$ are the power spectral densities (PSDs) of $u[n]$ and $v[n]$, respectively, and $c[n] \circ \longrightarrow C\left(e^{j \Omega}\right)$ is the Fourier transform of the channel impulse response. Note that the MMSE performance is influenced through the regularisation term in (1) by the signal-to-noise ratio (SNR) of the transmission system [7].

For the subband equaliser, we establish the hypothesis that aliased signal components can be treated as channel noise. In the $k$ th subband, we adopt the model depicted in Fig. 3. Source models $f_{1, k}[n]$ and $f_{2, k}[n]$ are utilised such that the sequences $u^{\prime}[n]$ and $v^{\prime}[n]$ are white Gaussian with PSDs $P_{u^{\prime} u^{\prime}}\left(e^{j \Omega}\right)=1$ and $P_{v^{\prime} v}\left(e^{j \Omega}\right)=1$. The source model $c_{k}[n]$ defines the unaliased channel component in the $k$ th subband, while $f_{1, k}[n]$ holds the passband and transition bands of the $k$ th analysis filter and any potential colouring of $u[n]$. Finally, the source model $f_{2, k}[n]$ in the noise path represents the aliased parts of the $k$ th analysis filter convolved with the channel. With the quantities defined in Fig. 3, the Wiener solution for the $k$ th subband equaliser, $W_{o p t, k}\left(e^{j \Omega}\right)$, can be written according to (1) as

$$
W_{\mathrm{opt}, k}\left(e^{j \Omega}\right)=\frac{\left|F_{1, k}\left(e^{j \Omega}\right)\right|^{2} \cdot C_{k}^{*}\left(e^{j \Omega}\right)}{\left|F_{1, k}\left(e^{j \Omega}\right)\right|^{2} \cdot\left|C_{k}\left(e^{j \Omega}\right)\right|^{2}+\left|F_{2, k}\left(e^{j \Omega}\right)\right|^{2}}
$$

In (2), $f_{1, k}[n] \circ \longrightarrow F_{1, k}\left(e^{j \Omega}\right)$ and $f_{2, k}[n] \circ \longrightarrow F_{2, k}\left(e^{j \Omega}\right)$ denote the Fourier transform of $f_{1, k}[n]$ and $f_{2, k}[n]$, respectively. Although not explicitly derived here, the reconstruction of the equivalent fullband Wiener filter $W_{\text {opt }}\left(e^{j \Omega}\right)$ can be (2) as a function of the analysis filter bank, the $K$ subband Wiener solutions $W_{o p t, k}\left(e^{j \Omega}\right)$, and the synthesis filter bank.

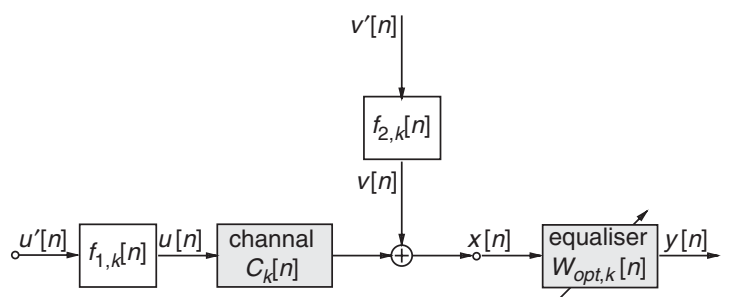

Fig. 3 Block diagram of Wiener filter $w_{\text {opt }, k}[n]$ for $k$ th subband adaptive equaliser

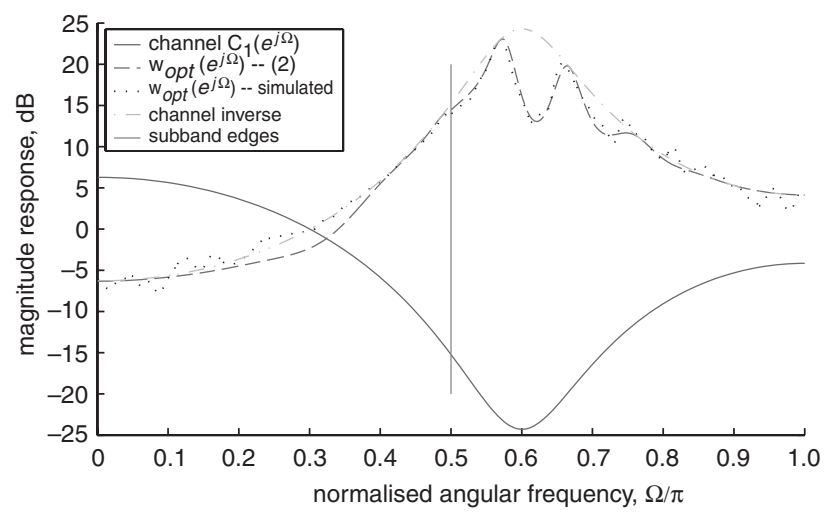

Fig. 4 Comparison between analytical and simulated results of $W_{\text {opt }}\left(e^{j \Omega}\right)$ for channel $C_{1}(z)$, using GDFT modulated filters with $K=4$ number of subband and decimated by factor of $N=3$

Simulation results: To compare the analytically derived Wiener solution with simulation results, no channel noise is added to isolate the limitations imposed by the subband structure. We consider a channel impulse response defined by its $z$-transform $C_{1}(z)=0.292+$ $0.360 z^{-1}+0.756 z^{-2}+0.360 z^{-3}+0.292 z^{-4}$ in combination with a subband equaliser operating in $K=4$ subbands decimated by $N=3$. The results are depicted in Fig. 4. The Figure shows a good match between the analytical calculation, $W_{o p t}\left(e^{j \Omega}\right)$ in (2), and the simulation result, $W_{\text {opt }}\left(e^{j \Omega}\right)$ simulated, for channel $C_{1}(z)$; a clear deviation from 
the zero-forcing solution owing to regularisation by aliasing can be observed on $W_{\text {opt }}\left(e^{j \Omega}\right)$. Therefore, we have verified the validity of our hypothesis to treat aliasing as channel noise by comparing the simulated answers with the derived Wiener solution for subband equalisers as discussed in the preceding Section.

Aliasing can be considered equivalent to channel noise, which permits quantifying its effect on the optimum solution. Since in a real application the equaliser would be prone to true channel noise, the regularisation term would be influenced by both aliasing and noise. Therefore, as long as the alias level of the subband equaliser remains below the injected channel noise, no MMSE limitations of the subband equaliser with respect to a fullband system are incurred. Thus, when designing a subband equaliser it is important to select the filter banks sufficient with respect to the channel SNR. Vice versa, the filter design only has to be good enough and therefore can be traded-off for, e.g., low delay properties.

Conclusion: We have demonstrated that the optimum subband adaptive equaliser is limited by aliasing owing to subband decimation, which as claimed in our hypothesis can be assumed to be equivalent to channel noise. This finding provides an important guideline for subband equaliser design with respect to achieving sufficient performance in a real situation. The effect of aliasing should be minimised below the channel SNR to guarantee satisfactory performance of subband adaptive systems.

(C) The Institution of Engineering and Technology 2006

15 June 2006

Electronics Letters online no: 20061890

doi: 10.1049/el:20061890
H. Mohamad (Faculty of Engineering, Multimedia University, Jalan Multimedia, Selangor, 63100 Cyberjaya, Malaysia)

E-mail: hafizal@mmu.edu.my

S. Weiss (Department of Electronics \& Electrical Engineering, University of Strathclyde, United Kingdom)

M. Rupp (Institute of Communications and Radio Frequency Engineering, TU Wein, Vienna, Austria)

L. Hanzo (School of Electronics \& Computer Science, University of Southampton, United Kingdom)

\section{References}

1 Qureshi, S.U.H.: 'Adaptive equalization', Proc. IEEE, 1985, 73, pp. $1349-1387$

2 Mohamad, H., Weiss, S., Rupp, M., and Hanzo, L.: 'Fast adaptation of fractionally spaced equalisers', Electron. Lett., 2002, 38, (2), pp. 96-98

3 Mohamad, H., Weiss, S., Mohd-Arif, N.A., and Alias, M.Y.: 'Subband decomposition techniques for adaptive channel equalisation'. Proc. 13th IEEE Int. Conf. on Networks, 2005, Vol. 2, pp. 617-621

4 Kellermann, W.: 'Analysis and design of multirate systems for the cancellation of acoustical echoes'. Proc. ICASSP, 1988, Vol. 3, pp. $2570-2573$

5 Weiss, S., Stenger, A., Stewart, R.W., and Rabenstein, R.: 'Steady-state performance limitations of subband adaptive filters', IEEE Trans. Signal Process., 2001, 49, (9), pp. 1982-1991

6 Girod, B., Rabenstein, R., and Stenger, A.: 'Signals and systems' (John Wiley \& Sons, 2001)

7 Casas, R.A., De-Victoria, F.L., Fijalkow, I., Schniter, P., Endrea, T.J., and Johnson, C.R., Jr.: 'On MMSE fractionally-spaced equalizer design'. Proc. 13th Int. Conf. on Signal Processing, 1997, Vol. 1, pp. 395-398 